Article

\title{
Single-Crystal Growth and Small Anisotropy of the Lower Critical Field in Oxypnictides: $\mathrm{NdFeAsO}_{1-x} \mathrm{~F}_{x}$
}

\author{
Abanoub R. N. Hanna ${ }^{1}$ (D) and Mahmoud Abdel-Hafiez ${ }^{1,2,3, *(D)}$ \\ 1 Institute of Physics, Goethe University Frankfurt, 60438 Frankfurt/M, Germany; \\ abanoub.hanna@helmholtz-Berlin.de \\ 2 Lyman Laboratory of Physics, Harvard University, Cambridge, MA 02138, USA \\ 3 Department of Physics and Astronomy, Box 516, Uppsala University, SE-75120 Uppsala, Sweden \\ * Correspondence: mhafiez@g.harvard.edu
}

Received: 7 April 2020; Accepted: 22 April 2020; Published: 1 May 2020

\begin{abstract}
High-quality single crystals of the unconventional superconductor $\mathrm{NdFeAsO}_{1-} \mathrm{F}_{x}$ were grown. We developed a new optimized flux technique to overcome the difficulties in single-crystal growth and the sample quality limitations of $\mathrm{NdFeAsO}_{1-x} \mathrm{~F}_{x}$. The normal state of the F-doped samples exhibits simple metallic behavior upon cooling down from room temperature, followed by a sharp superconducting transition. The values of residual resistivity ratio (RRR) is 3.2, 6.4, and 10.3 for $x=0.1,0.15$, and 0.2 , respectively. Both the large RRR and the narrow superconducting transition signpost the high quality of the crystals. We have examined the in- and out-of-plane lower critical fields, and the field at which vortices penetrate the sample of $\mathrm{NdFeAsO}_{1-x} \mathrm{~F}_{x}(x=0.1)$. The anisotropy ratio $\left[\gamma_{\mathrm{Hc} 1}(0)\right]$ increased slightly with increasing temperature from 0.8 Tc to Tc. The temperature dependence of the first vortex penetration field was obtained under the static magnetic field, $H$, parallel to the c- and ab- axis, and pronounced changes in the $H_{c 1}(T)$ curvature were observed, which are attributed to the multi-band superconductivity.
\end{abstract}

Keywords: crystal growth; unconventional superconductor; lower critical field

\section{Introduction}

In many correlated electron materials, several ground states are often competing with each other. Thus, adjusting certain parameters may allow switching from one ground state to another. Typical control parameters include the magnetic field, pressure, electron density, and chemical composition [1]. Searching for quaternary materials containing iron arsenide (FeAs) fluorite-type layers has brought new insights to the high- $\mathrm{T}_{\mathrm{C}}$ superconductivity community [2]. The $\mathrm{NdFeAsO}_{1-x} \mathrm{~F}_{x}$ compound is one of the 1111 iron pnictides: type-II superconductors containing sets of two-dimensional iron arsenide layers that exhibit a tetragonal structure at ambient conditions [3,4]. This compound shows a sharp superconducting (SC)transition [5]. Although the magnetic structure is the same as $\mathrm{LaFeAsO}$, an ordered magnetic moment of $0.25 \mu_{\mathrm{B}}$ was measured for this compound, which is one of the lowest observed in this type of material to date [6]. The resistivity measurements reveal quite a small anisotropy and a huge upper critical field, and thus, encourage several applications of this compound [7]. The detailed chemical composition of the $\mathrm{NdFeAsO}_{1-} \mathrm{F}_{x}$ samples with different $x$-values, as well as their electrical transport characterization, showed the emergence of superconductivity with fluorine doping at the level of 13-20\% [8]. Direct current (DC) magnetometry and muon-spin spectroscopy $(\mu \mathrm{SR})$ measurements revealed that, unlike the other Ln-1111 family members, in the intermediate F-doping regime of Nd-1111, there is a strong indication of fluctuating magnetism on the $\mu$ SR time scale [9]. Studying the magnetic structure of $\mathrm{Nd}$ in the parent compound $\mathrm{NdFeAsO}$ revealed a strong interplay between $\mathrm{Nd}$ and Fe moments at low temperatures $(\mathrm{T}=1.7-15 \mathrm{~K})$. Although $\mathrm{Nd}$ moments 
aligned along the crystallographic c-axis at a low temperature $(1.7 \mathrm{~K})$, increasing the temperature allowed the moment to reorient towards the crystallographic c-axis [10].

The main challenge in understanding unconventional superconductors is to have large and high-quality single-phase crystals. The lack of such samples has been a central impediment in resolving several issues in the field of unconventional superconductivity. For example, the structure of the gap function in the Brillouin zone is not known at a high resolution. Oxypnictides, $L n F e P n O(L n$ : lanthanide, $P n$ : pnictogen), abbreviated as 1111, are the most attractive materials among the discovered Fe-based superconductors due to their high SC transition temperatures, as well as their high upper critical fields, which make them suitable for high-field magnet applications. The main problems with the single-crystal growth of 1111 systems are the following [11]:

- The compounds decompose above $\sim 1200{ }^{\circ} \mathrm{C}$, well below the melting point;

- The presence of stable secondary phases (i.e., stable rare earth oxides compounds);

- The multicomponent phase diagrams are unknown.

Two main methods for the single-crystal growth of the 1111 compounds are as follows:

1. Growth by the flux method using metallic Tin (Sn), an equivalent mixture of sodium and potassium chlorides $(\mathrm{NaCl} / \mathrm{KCl})$, and iodides $(\mathrm{NaI} / \mathrm{KI})$, and sodium arsenide $(\mathrm{NaAs})$ as the flux. The following points were noticed:

- Sodium arsenide (NaAs) was shown to yield large and well-shaped single crystals [11]. However, the quality of these crystals is under debate. Unfortunately, F-doping was not yet possible using this flux;

- Tin (Sn) is a suitable flux for the $\mathrm{CeFe}\left(\mathrm{As}_{1-}{ }_{x} \mathrm{P}_{\mathrm{x}}\right) \mathrm{O}$ system, but not for the LaFeAsO compound [12];

- $\mathrm{NaI} / \mathrm{KI}$ flux was shown to give crystals with several $100 \mu \mathrm{m}$ lengths [13]. High temperatures need to be applied to increase the solubility of the elements in the salt flux.

2. High-pressure crystal growth using the $\mathrm{NaCl} / \mathrm{KCl}$ flux $[14,15]$. This method is favorable for the single-crystal growth of the 1111 systems. Using this technique, F-doped crystals were grown to crystal sizes below $500 \mu \mathrm{m}$. However, this is still too small for several experiments.

Among the widely studied superconducting 1111 compounds, a systematic investigation of the crossover region between the superconducting and the antiferromagnetic (AFM) phases for the $\mathrm{L} n=\mathrm{Nd}$, La, and Ce cases is missing. Sphinx will fill this gap by focusing on the growth of sizeable high-quality single crystals of $L n \mathrm{FeAsO}_{1-\mathrm{y}} \mathrm{F}_{\mathrm{x}}(\mathrm{Ln}=\mathrm{La}, \mathrm{Ce}, \mathrm{Nd})$ and $\operatorname{LnFe}\left(A s_{1-x} P n_{x}\right) O(P n=P, S b)$ compounds. These compounds have a relatively small number of publications so far. None of which address these important challenges concerning the mechanism of superconductivity, and there is no agreement on a generic electronic phase diagram as a function of chemical doping.

The flux growth method is one of the frequently used techniques for growing crystals of iron pnictides. High-temperature solution growth allows for a wide variety of flux materials and relatively low growth temperature, as well as a relatively small quantity of reactants, providing a new route for single-crystal growth from incongruently melting reactants. Although the flux growth method produces single crystals free from thermal strain, the produced crystals are relatively small. However, by optimizing the amount of flux, the temperature profile may increase the size and the quality of the product.

In this work, we report the growth of a relatively large single crystal of the $\mathrm{NdFeAsO}_{1-x} \mathrm{~F}_{x}$ compound with different nominal fluorine doping. These crystals were grown by the flux method using alkali metal chlorides $(\mathrm{NaCl} / \mathrm{KCl})$ eutectic flux. The superconducting properties of the prepared samples were investigated and indicated the appearance of diamagnetic properties. 


\section{Materials and Methods}

The growth of $\mathrm{NdFeAsO}_{1-x} \mathrm{~F}_{x}$ single crystals was done according to the previously reported method by Adamski et al. [16,17], with some modifications on both the flux stoichiometry and the temperature profile. Taking into consideration the safety precautions for handling arsenic, all procedures related to the sample preparation were performed under an argon atmosphere. The starting materials were neodymium metal (Nd), arsenic metal (As), iron (III) oxide $\left(\mathrm{Fe}_{3} \mathrm{O}_{4}\right)$, iron fluoride $\left(\mathrm{FeF}_{2}\right)$, potassium chloride $(\mathrm{KCl})$, and sodium chloride $(\mathrm{NaCl})$. The purity of the materials was $99.9 \%$ and was used without further purification. These materials were cut, weighed, and mixed inside an Argon filled glove box under a controlled level of $\mathrm{O}_{2}$ and $\mathrm{H}_{2} \mathrm{O}(0.5 \mathrm{ppm})$. The molar ratio of reactants and the flux were modified to be 1:10, instead of 1:7 in the previously reported data.

Figure 1 summarizes the crystal growth process. The mixture of the precursors and the flux were added to a glassy carbon crucible $(20 \mathrm{~mm})$ with a lid. To decrease the oxidation of the sample, the crucible was inserted into a niobium ampoule and covered. The ampoule was then sealed using an arc-welding machine. Finally, the whole sample was sealed under a vacuum inside a quartz tube using a quartz welding station and then transferred into a box furnace. For the protection of the furnace, the samples were kept inside a stainless-steel shield with a cover.

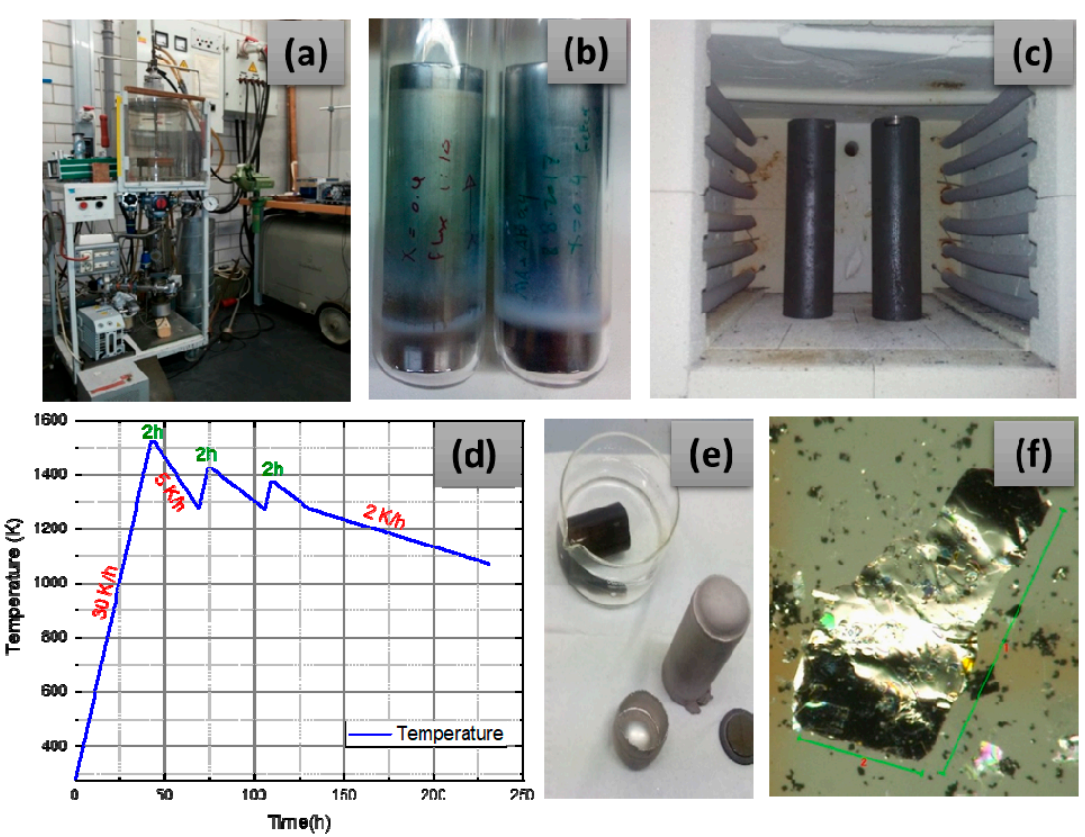

Figure 1. The tools used for the crystal growth process. (a) The argon arc machine used for wielding the niobium ampoule. (b) The quartz tube containing the sample sealed under vacuum. (c) The box furnace with a stainless-steel shield containing the sample. (d) The temperature profile for the crystal growth with multistep crystallization. (e) The removal of the flux using distilled water. (f) An optical image of one of the produced crystals $\left(800 \times 350 \mu \mathrm{m}^{2}\right)$.

The development of a suitable profile was quite challenging. The analysis of the powder diffraction patterns indicated that $\mathrm{NdFeAsO}_{1-x} \mathrm{~F}_{x}$ is a major phase, which means that the flux can dissolve most of the precursors. However, some relatively large and pure crystals were obtained. Most of the previous growth experiments using the flux method used a single step for crystallization which produced a large number of small-sized crystals. Since we could not increase temperature due to the decomposition of the compound, we tried to develop a way to increase both the nucleation and the growth time without increasing the temperature. The multistep temperature profile enhanced the crystal size. On one hand, heating the mixture after crystallization allowed the dissolving of smaller seeds formed in the first step, thus decreasing the number of possible crystallization sites. On the other hand, during the 
growth phase, melting can crystallize a smaller number of relatively large seeds which increases the size of the final crystal.

The growth process was carried out by applying a modified temperature profile using the Sawtooth profile, as shown in Figure 1d. In this profile, the sample was introduced at three crystalization steps at 1523, 1423, and $1373 \mathrm{~K}$, respectively, with a dwell time of $2 \mathrm{~h}$ each. The sample was then cooled gradually to $1073 \mathrm{~K}$ at $2 \mathrm{~K} / \mathrm{h}$ and then cooled rapidly to room temperature. The ampoule was cut using a stainless-steel cutting knife and the flux was dissolved by rinsing with distilled water. The crystals were separated by filtration and checked using an optical microscope. Relatively large single crystals were found $\left(800 \times 350 \mu \mathrm{m}^{2}\right)$, compared to the previously reported crystals produced using the same technique $\left(250 \times 250 \mu \mathrm{m}^{2}\right)$. X-ray powder diffraction patterns $(\mathrm{XRD})$ were collected at ambient conditions using a Siemens D 500 diffractometer with $2 \theta$ in the range of 20-80 degrees. Electron probe microanalysis (EPMA) was carried out using a Jeol JX $8900 \mathrm{R}$ microprobe equipped with four different probes. Both magnetic and transport measurements were carried out using a quantum design physical property measurement system (PPMS) instrument at the Institute of Physics, Goethe University Frankfurt. The measurements were carried out in the range between 2 and $300 \mathrm{~K}$ under different applied magnetic fields. The temperature dependence of the magnetic susceptibility, $\chi$, and isothermal magnetization, $\mathrm{M}$, versus the magnetic field, $\mathrm{H}$, measurements were performed both in-plane and out-of-plane, parallel to the applied field. The transport measurements were measured by making four gold leads $(10 \mu \mathrm{m})$ using an Epo-Tek H20E silver epoxy.

\section{Results and Discussion}

\subsection{Crystal Quality}

The purity phase of the single crystals and the exact amount of the fluorine dopant were confirmed by both EPMA and powder X-ray diffraction. The electron probe microanalyzer (EPMA) determined the exact amount of fluorine and oxygen in each crystal. Figure 2 represents the powder diffraction patterns of the different compounds. The diffraction patterns indicated a slight change in the lattice parameters and a regular shift in the Bragg peaks with different doping concentrations. These changes are attributed to the change in fluorine content as a result of replacing oxygen in the lattice, according to the previously reported data by Ponce-Flores and Conde-Gallardo [8].

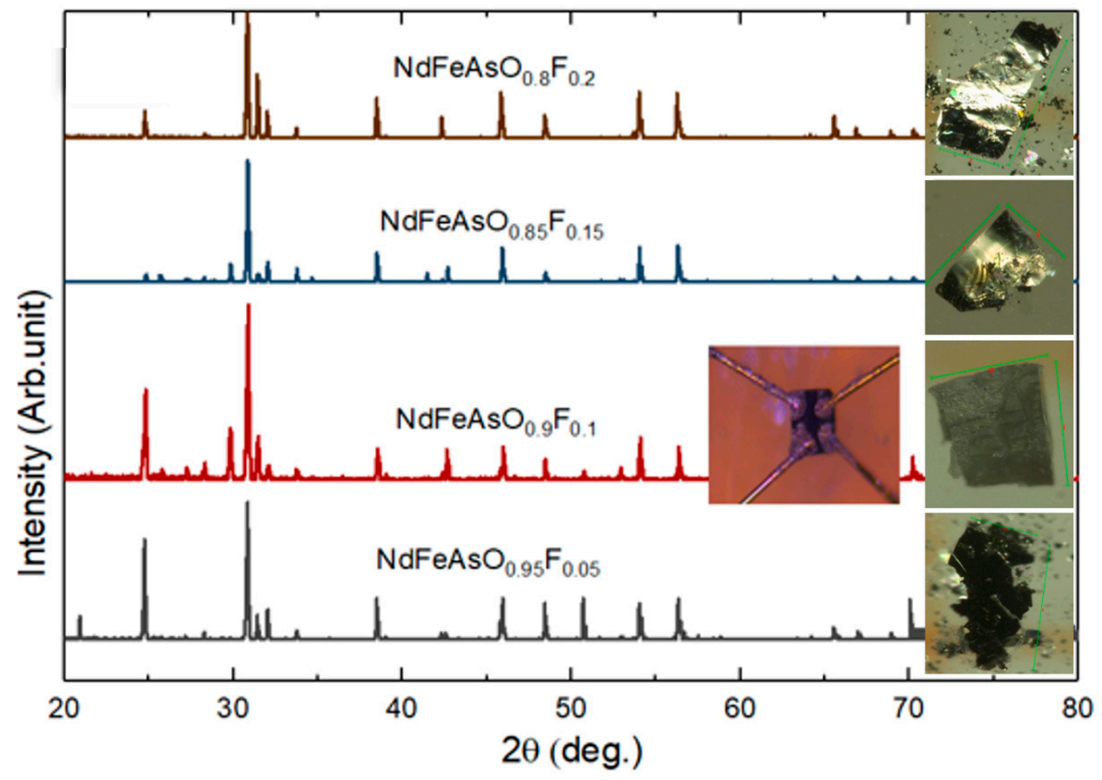

Figure 2. Powder $\mathrm{XRD}$ patterns for $\mathrm{NdFeAsO}_{1-x} \mathrm{~F}_{x}$ with different fluorine doping, together with a piece of crystal sample of each grown system. The inset is an image of a crystal with four probes connected on the surface for transport measurements. 


\subsection{Transport Properties}

Figure 3a elucidates the temperature dependence of the in-plane resistance measurements upon heating the $\mathrm{NdFeAsO}_{1-} \mathrm{F}_{x}$ single crystals. Obviously, in the undoped $\mathrm{NdFeAsO}$ system, the anomaly at $142 \mathrm{~K}$ in resistance measurements attributed to the phase transition from tetragonal $P 4 / \mathrm{nmm}$ to orthorhombic Cmma. The second anomaly found at $T_{N} \approx 130 \mathrm{~K}$ corresponds to the onset of the well-known spin-density-wave (SDW) stripe-like Fe order [18]. In the absence of an externally applied field, the doped samples show a typical cooldown resistance curve for $\mathrm{NdFeAsO}_{1-x} \mathrm{~F}_{x}(x=0.1,0.15$, and 0.2$)$ single crystals. Both the spin density waves and the structural transition have been suppressed upon increasing the doping. In all doping samples, one can see that the normal state exhibits a simple metallic behavior upon cooling down to room temperature, followed by a sharp SC transition at Tc $45.4,50.4$ and $52.8 \mathrm{~K}$ ( $90 \%$ of the normal state resistivity) for $x=0.1,0.15$ and 0.2 , respectively, shown in Figure 2b. The values of residual resistivity ratio (RRR) is 3.2, 6.4 and 10.3 for $x=0.1,0.15$ and 0.2 , respectively. Both the large RRR and narrow superconducting transition confirm the good quality of the samples investigated here.
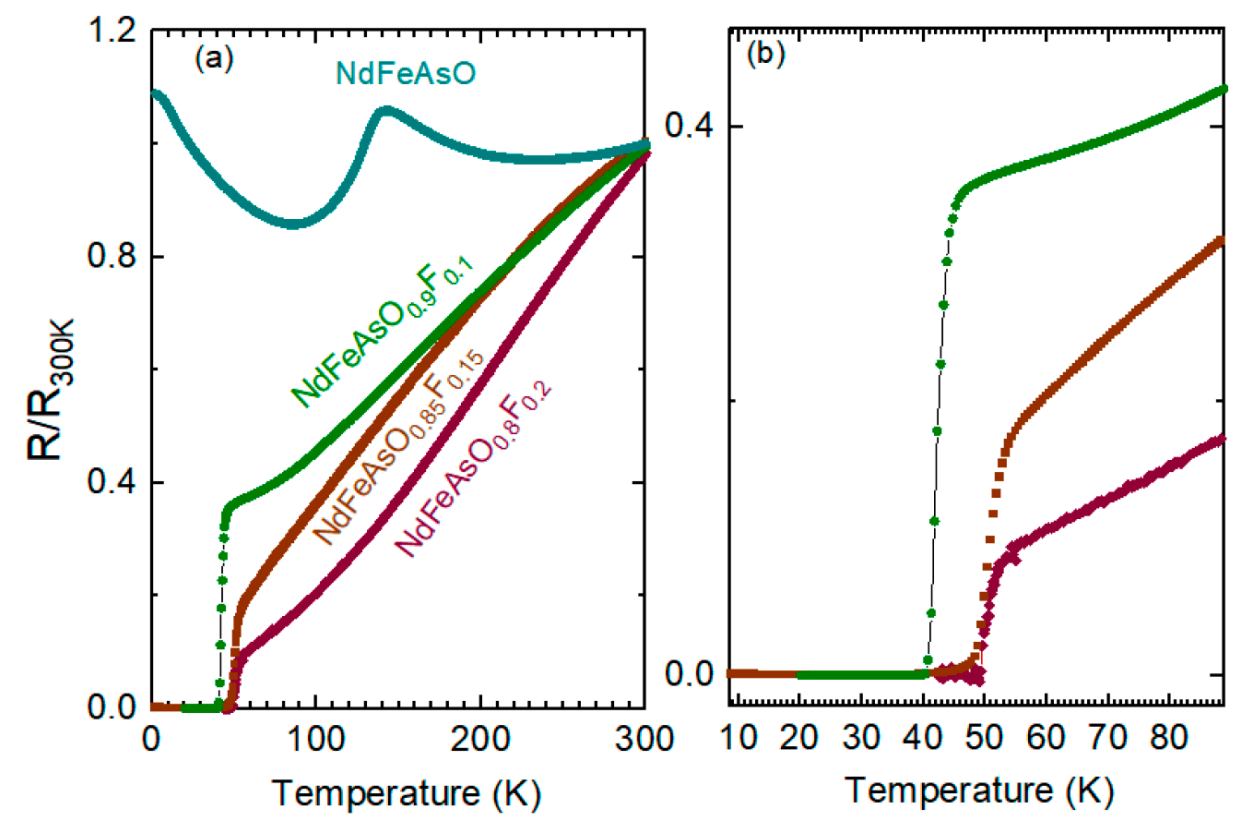

Figure 3. The transport measurements: (a) Illustrates the temperature dependence of the in-plane resistance measurements upon the heating of the $\mathrm{NdFeAsO}_{1-{ }_{x}} \mathrm{~F}_{x}$ single crystals at $\mathrm{x}=0,0.1,0.15$, and 0.2. (b) The close-up of the low-temperature region, highlighting the superconducting transition.

\subsection{AC Measurements}

Figure 4 represents the temperature dependence of the complex ac-susceptibility components of $\mathrm{NdFeAsO}{ }_{0.8} \mathrm{~F}_{0.2}$. The measurement was done in $5 \mathrm{Oe} a c$ field amplitude and $1 \mathrm{kHz}$ field frequency. The data were collected upon warming in the different dc magnetic fields after cooling in a zero magnetic field. The inset in Figure 4 presents the magnetic phase diagram of the temperature dependence of the upper critical field of $\mathrm{NdFeAsO}_{0.8} \mathrm{~F}_{0.2}$. The data were fitted according to the Werthamer-Helfand-Hohenberg (WHH) theory (solid line) [16]. The WHH theory predicts the behavior of $H_{c 2}(T c)$ by considering paramagnetic and orbital pair breaking. The investigated sample indicated a very high upper critical field, $H_{c 2}$. Using a fit to the data in the whole measurement range for negligible spin-paramagnetic effects $(\alpha=0)$ and spin-orbit scattering $(\lambda=0)$ yielded $\mu_{0} H_{c 2}=95 \mathrm{~T}$ for $x=0.2$. [18]. 


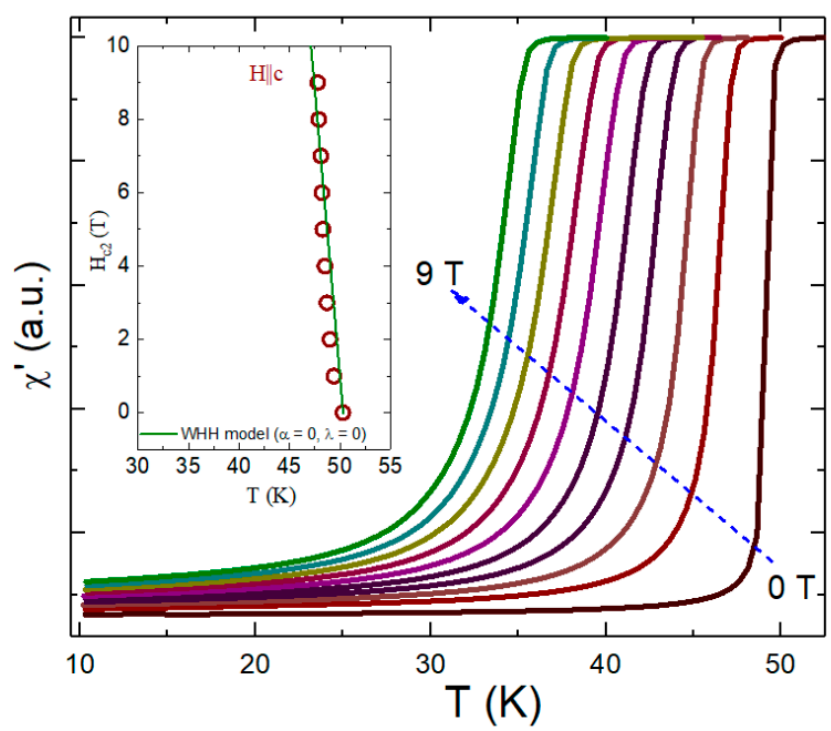

Figure 4. The temperature dependence of the complex ac-susceptibility components of $\mathrm{NdFeAsO}_{0.8} \mathrm{~F}_{0.2}$. The inset illustrates the phase diagram of $H_{c 2}$ vs. temperature. The solid lines are fits to the Werthamer-Helfand-Hohenberg (WHH) model for $\lambda=0$ with $\alpha=0$.

\subsection{Experimental Determination of the Lower Critical Field}

The lower critical field, $\mathrm{H}_{\mathrm{c} 1}$, is a valuable parameter giving essential information about the bulk thermodynamic properties of the material [19-22]. The lower critical field, $\mathrm{H}_{\mathrm{c} 1}$, was reliably determined from the magnetization measurements using two independent methods [16]. Once we experimentally obtained the values of $\mathrm{H}_{\mathrm{c} 1}$, we corrected them by accounting for the demagnetization effects. The low-temperature section of Figure 5 shows the flat behavior and the temperature dependence of the first vortex penetration field of both orientations. This shows a pronounced change of the $H_{c 1}(T)$ curvature, which is attributed to the multi-band superconductivity [16]. The inset in Figure 5 shows that the anisotropy of $\mathrm{H}_{\mathrm{c} 1}: \gamma_{\mathrm{Hc} 1}=\mathrm{H}_{\mathrm{c} 1}\left\|\mathrm{c} / \mathrm{H}_{\mathrm{c} 1}\right\| \mathrm{ab}$ has a small value. Similar behaviors have been reported in $\mathrm{LiFeAs}$ [23], $\mathrm{MgB}_{2}$ [24], and $\mathrm{PrFeAsO}_{1-\mathrm{y}}$ [25]. Such behaviors indicate a signature of multiband superconductivity. This could be due to the presence of Fermi surfaces of different sizes [26].

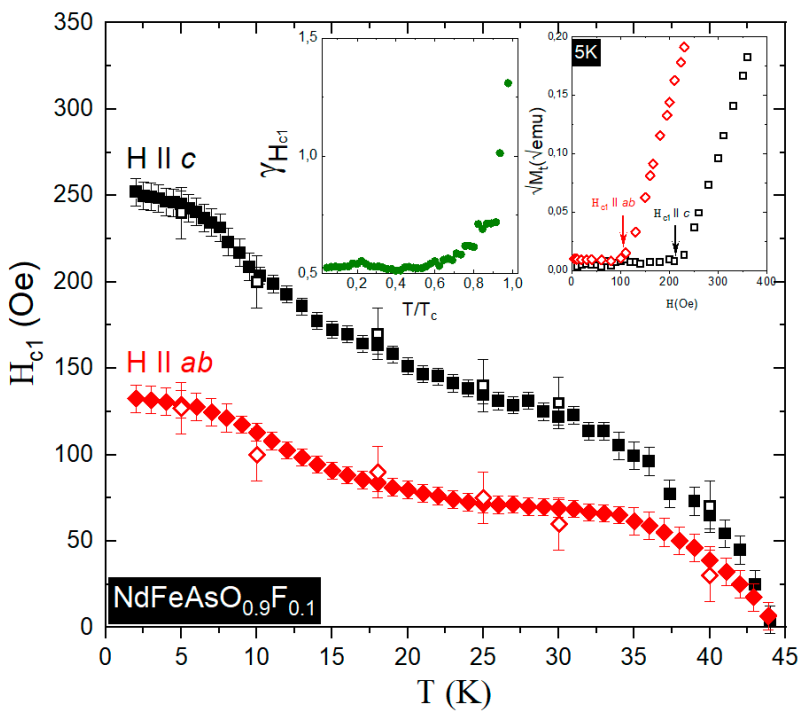

Figure 5. The extracted lower critical field, $\mathrm{H}_{\mathrm{c} 1}$, of $\mathrm{NdFeAsO}_{0.9} \mathrm{~F}_{0.1}$ as a function of temperature. The data were collected for both orientations for $\mathrm{H} \| a b$ and $\mathrm{H} \| c$. The bars show the uncertainty of estimation by the deviating point of the $\mathrm{M}-\mathrm{H}$ [11]. The inset shows the temperature dependence of the anisotropy of $\mathrm{H}_{\mathrm{c} 1}: \gamma_{\mathrm{Hc} 1}=\mathrm{H}_{\mathrm{c} 1}\left\|\mathrm{c} / \mathrm{H}_{\mathrm{c} 1}\right\| \mathrm{ab}$. 


\section{Conclusions}

In summary, we developed a new optimized flux technique to overcome the difficulties in single-crystal growth and the limitation of the sample quality of $\mathrm{NdFeAsO}_{1-x} \mathrm{~F}_{x}$. The temperature dependence of the first vortex penetration field was experimentally obtained by two independent methods, and the corresponding lower critical field was deduced. This study confirmed the small anisotropy ratio of the lower critical fields in $\mathrm{NdFeAsO}_{0.9} \mathrm{~F}_{0.1}$. The results were consistent with those reported previously.

Author Contributions: A.R.N.H. methodology, writing—original draft preparation. Crystal growth. M.A.-H. transport and magnetic measurements, writing-review and editing, supervision, project administration. All authors have read and agreed to the published version of the manuscript.

Funding: M.A. acknowledges the support from the Swedish Research Council (VR) 2018-05339 and the National Science Foundation (NSF).

Acknowledgments: The authors hereby acknowledge Cornelius Krellner for his contribution by providing access to the instruments at the AG Kellner research group. MA. acknowledges the support of AGYA (www.agya.info).

Conflicts of Interest: The authors declare no conflict of interest. The funders had no role in the design of the study; in the collection, analyses, or interpretation of data; in the writing of the manuscript, or in the decision to publish the results.

\section{References}

1. Coleman, P.; Schofield, A.J. Quantum Criticality. Nature 2005, 433, 226-229. [CrossRef] [PubMed]

2. Löhneysen, H.v.; Rosch, A.; Vojta, M.; Wölfle, P. Fermi-liquid instabilities at magnetic quantum phase transitions. Rev. Mod. Phys. 2007, 79, 1015-1075. [CrossRef]

3. Alborzi, Z.; Daadmehr, V. Synthesis and characterization of iron-based superconductor Nd-1111. Physica C: Supercond. 2018, 549, 116-118. [CrossRef]

4. Tapia-Ignacio, C.; Ponce-Flores, H.; Corrales-Mendoza, I.; Conde-Gallardo, A. Superconductivity, and Paramagnetism in the Nd-Based 1111 Oxypnictide Compounds. IEEE Trans. Appl. Supercond. 2017, 27, 1-5. [CrossRef]

5. Kito, H.; Eisaki, H.; Iyo, A. Superconductivity at $54 \mathrm{~K}$ in F-Free $\mathrm{NdFeAsO}_{1-\mathrm{y}}$. J. Phys. Soc. Jpn. 2008, 77, 063707. [CrossRef]

6. McGuire, M.A.; Hermann, R.P.; Sefat, A.S.; Sales, B.C.; Jin, R.; Mandrus, D.; Grandjean, F.; Long, G.J. Influence of the rare-earth element on the effects of the structural and magnetic phase transitions in $\mathrm{CeFeAsO}, \mathrm{PrFeAsO}$ and NdFeAsO. New J. Phys. 2009, 11, 025011. [CrossRef]

7. Jia, Y.; Cheng, P.; Fang, L.; Luo, H.; Yang, H.; Ren, C.; Shan, L.; Gu, C.; Wen, H.-H. Critical fields and anisotropy of $\mathrm{NdFeAsO}_{0.82} \mathrm{~F}_{0.18}$ single crystals. Appl. Phys. Lett. 2008, 93, 032503. [CrossRef]

8. Ponce-Flores, H.; Conde-Gallardo, A. Influence of the Oxygen Excess in the Synthesis of $\mathrm{NdFeAsO}_{1-x} \mathrm{~F}_{x}$ Superconductors. J. Supercond. Nov. Magn. 2013, 27, 673-679. [CrossRef]

9. Lamura, G.; Shiroka, T.; Bonfà, P.; Sanna, S.; De Renzi, R.; Putti, M.; Zhigadlo, N.D.; Katrych, S.; Khasanov, R.; Karpinski, J. Slow magnetic fluctuations and superconductivity in fluorine-doped NdFeAsO. Phys. Rev. B 2015, 91, 024513. [CrossRef]

10. Tehrani, F.S.; Daadmehr, V. The Superconductivity Mechanism in Nd-1111 Iron-Based Superconductor Doped by Calcium. J. Low Temp. Phys. 2020, 1-15. [CrossRef]

11. Yan, J.-Q.; Jensen, B.; Dennis, K.W.; McCallum, R.W.; Lograsso, T.A. Flux requirements for the growth of RFeAsO (R=rare earth) superconductors. Appl. Phys. Lett. 2011, 98, 072504. [CrossRef]

12. Krellner, C.; Geibel, C. Single-crystal growth and anisotropy of CeRuPO. J. Cryst. Growth 2008, 310, 1875-1880. [CrossRef]

13. Nitsche, F.; Jesche, A.; Hieckmann, E.; Doert, T.; Ruck, M. Structural trends from a consistent set of single-crystal data of RFeAsO (R=La, Ce, Pr, Nd, Sm, Gd, and Tb). Phys. Rev. B 2010, 82, 134514. [CrossRef]

14. Zhigadlo, N.D.; Katrych, S.; Bukowski, Z.; Weyeneth, S.; Puzniak, R.; Karpinski, J. Single crystals of superconducting SmFeAsO1 - xFygrown at high pressure. J. Phys. Condens. Matter 2008, 20, 342202. [CrossRef] 
15. Karpinski, J.; Zhigadlo, N.D.; Katrych, S.; Bukowski, Z.; Moll, P.; Weyeneth, S.; Keller, H.; Puzniak, R.; Tortello, M.; Daghero, D.; et al. Single crystals of LnFeAsO1 - xFx (Ln=La, Pr, Nd, Sm, Gd) and Ba1 xRbxFe2As2: Growth, structure and superconducting properties. Physica C: Supercond. 2009, 469, 370-380. [CrossRef]

16. Adamski, A.; Krellner, C.; Abdel-Hafiez, M. Signature of multigap nodeless superconductivity in fluorine-doped NdFeAsO. Phys. Rev. B 2017, 96, 100503. [CrossRef]

17. Adamski, A.; Nassief, A.R.; Krellner, C.; Abdel-Hafiez, M. Vortex dynamics and the critical current density in NdFeAsO0.9F0.1 single crystal. J. Magn. Magn. Mater. 2018, 459, 301-304. [CrossRef]

18. Abdel-Hafiez, M.; Mito, M.; Shibayama, K.; Takagi, S.; Ishizuka, M.; Vasiliev, A.N.; Krellner, C.; Mao, H.K. High-pressure phase diagram of $\mathrm{NdFeAsO}_{0.9} \mathrm{~F}_{0.1}$ : Disappearance of superconductivity on the verge of ferromagnetism from Nd moments. Phys. Rev. B 2018, 98, 094504. [CrossRef]

19. Abdel-Hafiez, M.; Ge, J.; Vasiliev, A.N.; Chareev, D.A.; Van de Vondel, J.; Moshchalkov, V.V.; Silhanek, A.V. Temperature dependence of lower critical field $\mathrm{H}_{\mathrm{c} 1}(\mathrm{~T})$ shows nodeless superconductivity in FeSe. Phys. Rev. B 2013, 88, 174512. [CrossRef]

20. Abdel-Hafiez, M.; Zhang, Y.-Y.; Cao, Z.-Y.; Duan, C.-G.; Karapetrov, G.; Pudalov, V.M.; Vlasenko, V.A.; Sadakov, A.V.; Knyazev, D.A.; Romanova, T.A.; et al. Superconducting properties of sulfur-doped iron selenide. Phys. Rev. B 2015, 91, 165109. [CrossRef]

21. Abdel-Hafiez, M.; Pereira, P.J.; Kuzmichev, S.A.; Kuzmicheva, T.E.; Pudalov, V.M.; Harnagea, L.; Kordyuk, A.A.; Silhanek, A.V.; Moshchalkov, V.V.; Shen, B.; et al. Lower critical field and SNS-Andreev spectroscopy of 122-arsenides: Evidence of nodeless superconducting gap. Phys. Rev. B 2014, 90, 054524. [CrossRef]

22. Abdel-Hafiez, M.; Zhao, Y.; Huang, Z.; Cho, C.W.; Wong, C.H.; Hassen, A.; Ohkuma, M.; Fang, Y.W.; Pan, B.J.; Ren, Z.A.; et al. High-pressure effects on isotropic superconductivity in the iron-free layered pnictide superconductor $\mathrm{BaPd}_{2} \mathrm{As}_{2}$. Phys. Rev. B 2018, 97, 134508. [CrossRef]

23. Song, Y.J.; Ghim, J.S.; Yoon, J.H.; Lee, K.J.; Jung, M.H.; Ji, H.-S.; Shim, J.H.; Bang, Y.; Kwon, Y.S. Small anisotropy of the lower critical field and the $\mathrm{s} \pm$-wave two-gap feature in single-crystal LiFeAs. EPL (Europhysics Letters) 2011, 94, 57008. [CrossRef]

24. Lyard, L.; Szabó, P.; Klein, T.; Marcus, J.; Marcenat, C.; Kim, K.H.; Kang, B.W.; Lee, H.S.; Lee, S.I. Anisotropies of the Lower and Upper Critical Fields in $\mathrm{MgB}_{2}$ Single Crystals. Phys. Rev. Lett. 2004, 92, 057001. [CrossRef]

25. Hashimoto, K.; Shibauchi, T.; Kato, T.; Ikada, K.; Okazaki, R.; Shishido, H.; Ishikado, M.; Kito, H.; Iyo, A.; Eisaki, H.; et al. Microwave Penetration Depth and Quasiparticle Conductivity of $\mathrm{PrFeAsO}_{1-y}$ Single Crystals: Evidence for a Full-Gap Superconductor. Phys. Rev. Lett. 2009, 102, 017002. [CrossRef]

26. Singh, D.J. Electronic structure and doping in $\mathrm{BaFe}_{2} \mathrm{As}_{2}$ and LiFeAs: Density functional calculations. Phys. Rev. B 2008, 78, 094511. [CrossRef] 